\title{
High-harmonic generation in solids using a mid-infrared sub-cycle pulse synthesizer
}

\author{
Kyung-Han Hong ${ }^{1, *}$, Zhou Wang ${ }^{2}$, Tobias Kroh ${ }^{1,3}$, Hyunwook Park ${ }^{2}$, Peter Krogen ${ }^{1}$, \\ Houkun Liang ${ }^{1}$, Louis F. DiMauro ${ }^{2}$, and Franz X. Kärtner ${ }^{1,3}$ \\ ${ }^{1}$ Department of Electrical Engineering and Computer Science and Research Laboratory of Electronics, \\ Massachusetts Institute of Technology (MIT), Cambridge, Massachusetts 02139, USA \\ ${ }^{2}$ Department of Physics, The Ohio State University, Columbus, Ohio 43210, USA \\ ${ }^{3}$ Center for Free-Electron Laser Science, DESY and Department of Physics, University of Hamburg, D-22607 Hamburg, Germany \\ *E-mail address: kyunghan@mit.edu
}

\begin{abstract}
We generate high-order harmonics in silicon using a mid-infrared (2.5-9.0 $\mu \mathrm{m})$ pulse synthesizer. Even and odd harmonics ( $19^{\text {th }}$ order) with few-cycle pulses and near-continuous spectra with sub-cycle pulses, indicating an isolated harmonic emission, are observed. OCIS codes: (320.7110) Ultrafast nonlinear optics; (270.6620) Strong-field processes
\end{abstract}

\section{Introduction}

High-energy, carrier-envelope phase (CEP)-stable, sub-cycle pulses can provide unique opportunities of exploring isolated phase-sensitive strong-field light-matter interactions in atoms, molecules and solids. At the mid-infrared (midIR) wavelength range the Keldysh parameter is much smaller than unity even at relatively modest laser intensities, enabling to study the strong-field sub-cycle electron dynamics in solids via high-harmonic generation (HHG) [1-3] without damage as well as the electron tunneling dynamics in nano-devices. These efforts are opening a great opportunity towards petahertz electronics. Regarding the generation of high-energy sub-cycle pulses, pulse synthesis is a very powerful method to overcome the bandwidth limit of conventional laser amplifiers. One can generate and manipulate an optical bandwidth more than one octave by coherently combining ultrashort pulses covering different spectral windows with controlled phase. Single- or sub-cycle pulse synthesizers in the visible-to-near-infrared (IR) spectral region have been demonstrated by coherent multiplexing of pulses from fiber lasers, supercontinuum [4], and optical parametric chirped-pulse amplifiers (OPCPA's) [5]. In this contribution, we present a multi-GW, mid-IR subcycle pulse synthesizer based on an optical parametric amplifier (OPA) and its application to HHG in solids.

\section{Mid-infrared sub-cycle pulse synthesizer}

We generate CEP-stable, $33 \mu \mathrm{J}$, 0.88-cycle mid-IR pulses from a $2.1-\mu \mathrm{m}$ pumped OPA by coherently synthesizing the passively CEP-stable few-cycle signal and idler pulses with the combined spectrum covering 2.5-9.0 $\mu \mathrm{m}$ [6]. In-line pulse synthesis is realized through a type-I collinear OPA with minimal temporal walk-off in a thin $\mathrm{CdSiP}_{2}$ (CSP) crystal. The CEP-stable signal pulse at 2.5-4.4 $\mu \mathrm{m}$ is produced via white-light generation of the CEP-stable $26 \mathrm{fs}$ pump pulse from a kHz, 2.1- $\mu \mathrm{m}$ OPCPA [7], while the passively CEP-stable idler pulse is obtained within the OPA process. The CSP crystal allows ultrabroad phase matching bandwidth of the idler covering 4.4-9.0 $\mu \mathrm{m}$ and the temporal walk-off between the signal pulse and idler is only $\sim 5 \mathrm{fs}$ with $1.1 \mathrm{~mm}$ of thickness. The near-Gaussian signal and idler beams are obtained and spatially overlapped. The temporal profile of the synthesized pulse is characterized with a cross-correlation frequency-resolved optical gating (XFROG) device, as presented in Figs. 1(a)-(d). The synthesized pulse has a near-transform-limited main peak and rippling wings, attributed to the interference of the signal and idler pulses. The synthesized pulse duration is measured as $\sim 12.4 \mathrm{fs}$ in FWHM centered at $\sim 4.2 \mu \mathrm{m}$, corresponding to 0.88 optical cycle. With the energy portion into the main pulse of $\sim 70 \%$ the peak power reaches $\sim 1.9$ GW. The shot-to-shot CEP fluctuations of signal and idler pulses are measured as $<300$ mrad over 6 minutes, characterized using $f$ - $3 f$ self-referencing and cross-referencing interferometry as shown in Figs. 1(e) and (f), respectively, ensuring the CEP stability of the synthesized pulses.

\section{HHG in solids}

We have performed HHG in solids, such as silicon (Si), ZnO, and NiO, using this mid-IR source. The HHG spectra with 200-nm and 500-nm-thick Si samples are found in Fig. 2. We have obtained discrete harmonics using few-cycle ( $43 \mathrm{fs}$ ) mid-IR pulses, linearly chirped in a 1-mm-thick Si plate, as shown in Figs. 2(a)-(c). Both even and odd harmonics up to the $\sim 19^{\text {th }}$ order of a $\sim 4.4 \mu \mathrm{m}$ center wavelength are obtained with the $\sim 43$-fs pulses because the twocolor driving field unbalances the electron trajectories and breaks the symmetry, whereas odd harmonics are observed with the signal pulses only. The intensity of the chirped pulse is estimated to be $\sim 2 \times 10^{12} \mathrm{~W} / \mathrm{cm}^{2}$ corresponding to an electric field strength of $0.4 \mathrm{~V} / \AA$. Fig. 2(c) shows the 4-fold symmetry around the Si $<100>$ axis, confirming that 
harmonics are produced from the crystal bands of Si. Finally, a near-continuous harmonic spectrum is observed when directly driven by the sub-cycle pulse, as shown by Fig. 2(d), which strongly indicates isolated harmonic emission. The CEP dependence along with a time-domain study could demonstrate the isolated sub-cycle control of electron dynamics in solids.
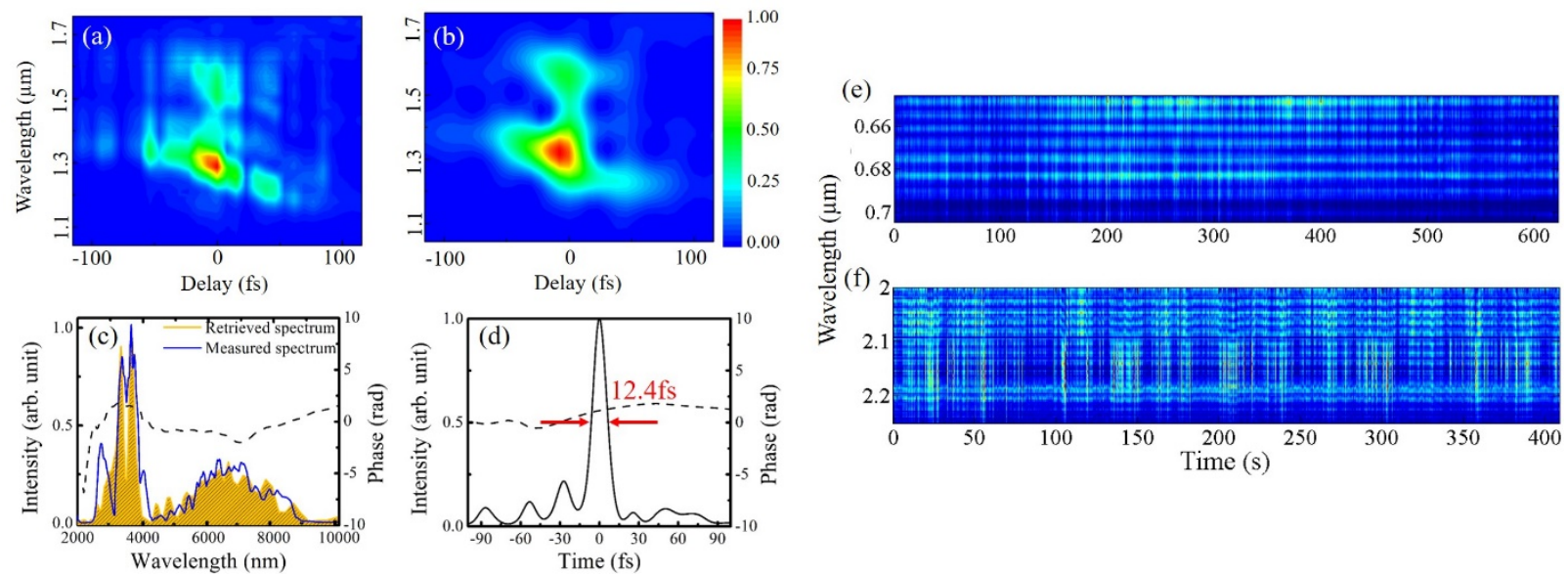

Fig. 1 The temporal characterization of the synthesized mid-IR pulse using XFROG and the measurement of shot-to-shot CEP stability of signal and idler pulses. The measured (a) and the retrieved (b) XFROG traces. The retrieved spectral (c) and temporal (d) intensity profiles of the synthesized pulse. The dotted curves are the retrieved phase. The $f$ - $3 f$ self-referencing interferometry of signal pulses (e) and the $f-3 f$ cross-referencing interferometry of idler pulses (f), showing the stable CEP over $>6$ minutes.
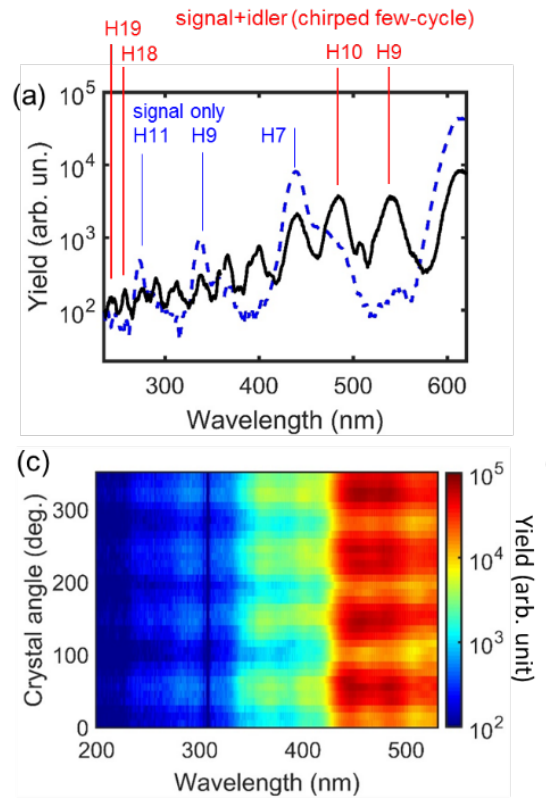

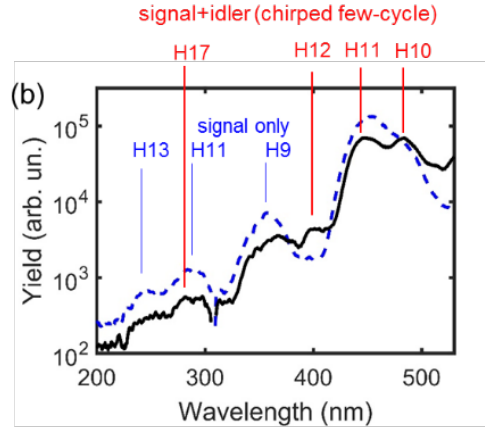

(d)

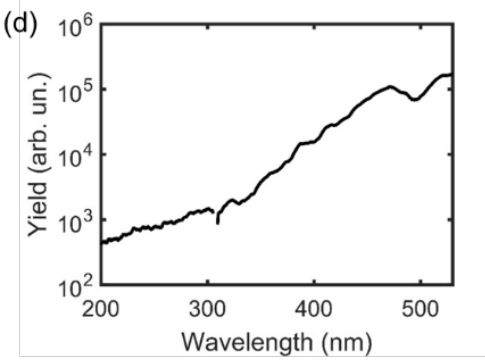

Fig. 2 Harmonic spectra generated in a 200nm-thick free-standing Si sample (a) and a 500-nm-thick Si sample on a 0.5 -mm-thick sapphire substrate (b), driven by chirped, $\sim 43$ fs pulses. The blue dotted line represents the spectrum generated by the signal pulse only. (c) The angle dependence of the harmonic spectra about the Si axis $<001>$ relative to the laser polarization. (d) The near-continuous harmonic spectrum generated by the synthesized sub-cycle pulse without chirping.

\section{References}

[1] S. Ghimire et al., “Observation of high-order harmonic generation in a bulk crystal,” Nature Phys. 7 138-141 (2011).

[2] O. Schubert et al., "Sub-cycle control of terahertz high-harmonic generation by dynamical Bloch oscillations," Nature Photon. 8, 119 (2014).

[3] G. Vampa et al., "Linking high harmonics from gases and solids," Nature 522, 462 (2015).

[4] A. Wirth et al., "Synthesized light transients," Science 334, 195-200 (2011).

[5] S.-W. Huang et al., "High-energy pulse synthesis with sub-cycle waveform control for strong-field physics," Nature Photon. 5, 475-479 (2011).

[6] H. Liang et al., "High-energy mid-infrared sub-cycle pulse synthesis from a parametric amplifier," Nature Comm., in press (2017).

[7] K.-H. Hong et al., "Multi-mJ, kHz, $2.1 \mu \mathrm{m}$ optical parametric chirped-pulse amplifier and high-flux soft x-ray high harmonic generation," Opt. Lett. 39, 3145-3148 (2014). 